\title{
Farnesyltransferase/Geranylgeranyltransferase Inhibitor L-778,123
}

National Cancer Institute

\section{Source}

National Cancer Institute. Farnesyltransferase/Geranylgeranyltransferase Inhibitor L778,123. NCl Thesaurus. Code C1840.

\begin{abstract}
A benzonitrile derivative capable of inhibiting some prenyltransferases. L-778,123 is a dual inhibitor of farnesyl:protein and geranylgeranyl:protein transferases; both enzymes catalyze prenylation of oncoprotein KRAS, a prerequisite step in activation of KRAS in signal transduction pathway of apoptosis. Although this agent was developed in part as an anti-KRAS agent, L-778,123 failed in a Phase I trial to inhibit KRAS, which is associated with many types of solid tumors.
\end{abstract}

